\title{
Global existence and exponential decay of strong solutions for the three-dimensional Boussinesq equations
}

\section{Zhaoyang Shang ${ }^{1,2^{*}}$ id and Fuquan Tang ${ }^{2}$}

\section{"Correspondence:}

zhaoyang.shang@163.com

'School of Finance, Shanghai Lixin

University of Accounting and

Finance, Shanghai, P.R. China

${ }^{2} S c h o o l$ of Mathematical Sciences,

Shanghai Jiao Tong University,

Shanghai, P.R. China

\section{Springer}

\begin{abstract}
In this paper, we consider the global existence of strong solutions to the three-dimensional Boussinesq equations on the smooth bounded domain $\Omega$. Based on the blow-up criterion and uniform estimates, we prove that the strong solution exists globally in time if the initial $L^{2}$-norm of velocity and temperature are small. Moreover, an exponential decay rate of the strong solution is obtained.
\end{abstract}

Keywords: Boussinesq equations; Blow-up criterion; Global strong solutions; Exponential decay rate

\section{Introduction}

In this paper, we consider the following three-dimensional incompressible Boussinesq equations in the Eulerian coordinates:

$$
\left\{\begin{array}{l}
u_{t}-\mu \Delta u+u \cdot \nabla u+\nabla P=\theta e_{3}, \\
\theta_{t}-\kappa \Delta \theta+u \cdot \nabla \theta=0, \\
\nabla \cdot u=0, \\
u(x, 0)=u_{0}(x), \quad \theta(x, 0)=\theta_{0}(x),
\end{array}\right.
$$

where $u=\left(u_{1}, u_{2}, u_{3}\right)(x, t), \theta=\theta(x, t), P(x, t)$ are unknown functions denoting fluid velocity vector field, absolute temperature, and scalar pressure, $t \geq 0$ is time, $x \in \Omega$ is spatial coordinate. $\mu$ is the kinematic viscosity, $\kappa$ is the thermal diffusivity, and $e_{3}=(0,0,1)$ is the unit vector in the $x_{3}$ direction. The given functions $u_{0}$ and $\theta_{0}$ are the initial velocity and initial temperature, respectively.

Boussinesq system (1.1) has been widely used in atmospheric sciences and oceanic fluids [9], and there is a huge amount of literature on the well-posedness theory of strong and week solutions for the three-dimensional Boussinesq equations. In 2004, Sawada and Taniuchi [12] established the local existence and uniqueness of strong solutions in the whole space. In 2008, Danchin and Paicu [6] obtained global small solutions in Lorentz spaces. In 2012, Brandolese and Schonbek [2] showed the polynomial decay rate of week

(c) The Author(s) 2020. This article is licensed under a Creative Commons Attribution 4.0 International License, which permits use, sharing, adaptation, distribution and reproduction in any medium or format, as long as you give appropriate credit to the original author(s) and the source, provide a link to the Creative Commons licence, and indicate if changes were made. The images or other third party material in this article are included in the article's Creative Commons licence, unless indicated otherwise in a credit line to the material. If material is not included in the article's Creative Commons licence and your intended use is not permitted by statutory regulation or exceeds the permitted use, you will need to obtain permission directly from the copyright holder. To view a copy of this licence, visit http://creativecommons.org/licenses/by/4.0/. 
and strong solutions, global smooth solution and its stability were given by Liu and Li [8] in 2014. In 2016, Qin et al. [11] established the global classical solutions for axisymmetric equations with anisotropic initial data. In 2017, Ye [15] proved global existence of smooth solution to a modified Boussinesq model without thermal diffusion. In 2018, Wen and Ye [14] established the regularity and uniqueness of strong solution for the damped Boussinesq equations with zero thermal diffusion. Global well-posedness with fractional partial dissipation can be found in a recent work [16]. For local and global theories of solutions in two-dimensional space, we refer to $[1,3-5,10,17]$ and the references therein.

Inspired by the results of full compressible Navier-Stokes equations [13] and nonhomogeneous incompressible magnetohydrodynamic equations [7], in this paper we consider global existence and exponential decay of strong solutions to system (1.1) with the following initial-boundary conditions:

$$
\begin{aligned}
& \left.(u, \theta)(x, t)\right|_{t=0}=\left(u_{0}(x), \theta_{0}(x)\right), \\
& \left.(u, \theta)(x, t)\right|_{\partial \Omega}=0 .
\end{aligned}
$$

Now, we state our main results as follows.

Theorem 1.1 (Blow-up criterion) Suppose that the initial data satisfy $\left(u_{0}, \theta_{0}\right) \in H_{0}^{1} \cap H^{2}$ and $\operatorname{div} u_{0}=0$ in $\Omega$. Let $(u, \theta)$ be the strong solution to the initial-boundary value problem (1.1)-(1.3) on $\Omega \times(0, T]$ satisfying

$$
\left\{\begin{array}{l}
(u, \theta) \in L^{\infty}\left(0, T ; H^{1}\right) \cap L^{2}\left(0, T ; H^{2}\right), \\
\left(u_{t}, \theta_{t}\right) \in L^{\infty}\left(0, T ; L^{2}\right) \cap L^{2}\left(0, T ; H^{1}\right) .
\end{array}\right.
$$

If $T^{*}<+\infty$ is the maximal time of existence for the strong solution $(u, \theta)$, then

$$
\lim _{T \rightarrow T^{*}} \sup _{[0, T)}\|\nabla u\|_{L^{\infty}\left(0, T ; L^{2}\right)}=+\infty .
$$

Theorem 1.2 (Global strong solution) For any given $K_{i}>0(i=1,2)$, suppose that the initial data satisfy $\left(u_{0}, \theta_{0}\right) \in H_{0}^{1} \cap H^{2}, \operatorname{div} u_{0}=0$ in $\Omega$, and

$$
\left\|\nabla u_{0}\right\|_{L^{2}} \leq \sqrt{K_{1}}, \quad\left\|\theta_{0}\right\|_{L^{2}} \leq \sqrt{K_{2}}
$$

Then the initial-boundary value problem (1.1)-(1.3) admits a unique global strong solution $(u, \theta)$ on $\Omega \times[0, T]$ for any $T>0$, provided that there exists a constant $\varepsilon_{0}>0$ such that

$$
\left\|u_{0}\right\|_{L^{2}}^{2}+\left\|\theta_{0}\right\|_{L^{2}}^{2} \leq \varepsilon_{0}
$$

where $\varepsilon_{0}$ depends on $K_{1}, \mu, \kappa$, and some other known constants but is independent of $T$.

Remark 1.1 When $\theta=0$, system (1.1) reduces to Navier-Stokes equations. Then Theorem 1.2 implies that Navier-Stokes equations admit a unique global strong solution on $\Omega \times[0, T]$ for any $T>0$, provided that there exists a constant $\varepsilon_{0}>0$ such that

$$
\left\|u_{0}\right\|_{L^{2}}^{2} \leq \varepsilon_{0}
$$

where $\varepsilon_{0}$ depends on $K_{1}, \mu$ and some other known constants but is independent of $T$. 
Theorem 1.3 (Asymptotic behavior) Under the conditions of Theorem 1.2, it holds that

$$
\int_{\Omega}\left(\theta^{2}+|\nabla u|^{2}\right) \leq C_{1} \exp \left\{-C_{2} t\right\}
$$

for any $t \in[0,+\infty)$, provided that there exists a constant $\varepsilon_{0}>0$ such that

$$
\left\|u_{0}\right\|_{L^{2}}^{2}+\left\|\theta_{0}\right\|_{L^{2}}^{2} \leq \varepsilon_{0}
$$

where positive constants $C_{1}, C_{2}, \varepsilon_{0}$ depend on $K_{1}, K_{2}, \mu, \kappa$, and some other known constants but are independent of $t$.

Remark 1.2 In Theorem 1.3, we obtain exponential decay rate (1.9) in a bounded domain, which refines the polynomial decay in [2] and [8].

For the fixed viscosity and heat conduction, we need the "smallness" of initial velocity and temperature. But the velocity and temperature can be large. From the proof of Theorem 1.2 and Theorem 1.3, we have the following corollary.

Corollary 1.4 For any given $K_{i}>0(i=1,2)$, suppose that the initial data satisfy $\left(u_{0}, \theta_{0}\right) \in$ $H_{0}^{1} \cap H^{2}, \operatorname{div} u_{0}=0$ in $\Omega$, and

$$
\left\|\nabla u_{0}\right\|_{L^{2}} \leq \sqrt{K_{1}}, \quad\left\|\theta_{0}\right\|_{L^{2}} \leq \sqrt{K_{2}}
$$

Then the initial-boundary value problem (1.1)-(1.3) admits a unique global strong solution $(u, \theta)$ on $\Omega \times[0, T]$ for any $T>0$, provided that there exists a constant $\mu^{*}>0$ such that

$$
\mu \geq \mu^{*}
$$

Furthermore, when $\mu$ and $\kappa$ are large enough, the global strong solution satisfies the following exponential decay rate:

$$
\int_{\Omega}\left(\theta^{2}+|\nabla u|^{2}\right) \leq C_{1} \exp \left\{-C_{2} t\right\}
$$

for any $t \in[0,+\infty)$, where positive constants $C_{1}, C_{2}, \mu^{*}$ depend on $K_{1}, K_{2}, \mu, \kappa$, and some other known constants but are independent of $t$.

Notations In the following paragraph, positive generic constants are denoted by $C$, which may change in different places.

The rest of the paper is organized as follows. In Sect. 2, under the assumption of (1.5) is false, we prove that the maximal time of existence for the strong solution is $T^{*}=+\infty$, where the standard energy estimate is used and it holds uniform in time. In Sect. 3, we show that $\|\nabla u\|_{L^{\infty}\left(0, T ; L^{2}\right)}$ will never blow up in finite time, which combines the blow-up criterion in Theorem 1.1, global existence of strong solution is proved in Theorem 1.2 provided the initial data of velocity and temperature are suitably small under the $L^{2}$-norm. Finally, in Sect. 4, exponential decay rate of the strong solution is obtained. 


\section{Blow-up criterion}

Now, we state some uniform a priori estimates to prove Theorem 1.1.

Lemma 2.1 Under the conditions of Theorem 1.1, it holds that

$$
\begin{aligned}
& \sup _{0 \leq t \leq T}\|\theta(t)\|_{L^{2}}^{2}+\int_{0}^{T} \kappa\|\nabla \theta(s)\|_{L^{2}}^{2} d s=\left\|\theta_{0}\right\|_{L^{2}}^{2} \leq K_{2}, \\
& \sup _{0 \leq t \leq T}\|u(t)\|_{L^{2}}^{2}+\int_{0}^{T} \mu\|\nabla u(s)\|_{L^{2}}^{2} d s \leq\left\|u_{0}\right\|_{L^{2}}^{2}+\frac{4}{\mu \kappa}\left\|\theta_{0}\right\|_{L^{2}}^{2} \leq K_{1}+\frac{4 K_{2}}{\mu \kappa} .
\end{aligned}
$$

Proof Multiplying temperature equation $(1.1)_{2}$ by $\theta$ integration by part and using divergence free property, we get

$$
\frac{1}{2} \frac{d}{d t}\|\theta(t)\|_{L^{2}}^{2}+\kappa\|\nabla \theta(s)\|_{L^{2}}^{2}=0
$$

integrating $(2.3)$ over $(0, t),(2.1)$ is proved.

Multiplying momentum equation $(1.1)_{1}$ by $u$ and using the Poicaré inequality, we obtain

$$
\frac{1}{2} \frac{d}{d t}\|u(t)\|_{L^{2}}^{2}+\mu\|\nabla u\|_{L^{2}}^{2} \leq\|u\|_{L^{2}}\|\theta\|_{L^{2}} \leq \frac{\mu}{2}\|\nabla u\|_{L^{2}}^{2}+\frac{2}{\mu}\|\nabla \theta\|_{L^{2}}^{2} .
$$

Then we have

$$
\frac{d}{d t}\|u(t)\|_{L^{2}}^{2}+\mu\|\nabla u\|_{L^{2}}^{2} \leq \frac{4}{\mu}\|\nabla \theta\|_{L^{2}}^{2}
$$

Integrating the above inequality over time $(0, t)$ and combining $(2.1)$, one can get $(2.2)$.

Lemma 2.2 Under the conditions of Theorem 1.1, and suppose (1.5) is false. Then it holds that

$$
\sup _{0 \leq t \leq T}\left(\|\nabla u(t)\|_{L^{2}}^{2}+\|\nabla \theta(t)\|_{L^{2}}^{2}\right)+\int_{0}^{T}\left(\left\|u_{t}(s)\right\|_{L^{2}}^{2}+\left\|\nabla^{2} \theta(s)\right\|_{L^{2}}^{2}\right) d s \leq C,
$$

where constant $C$ depends on the initial data, $\mu, \kappa$, and some other known constants but is independent of $T$.

Proof Multiplying momentum equation $(1.1)_{1}$ by $u_{t}$ and integrating by parts, we have

$$
\begin{aligned}
\frac{\mu}{2} \frac{d}{d t}\|\nabla u(t)\|_{L^{2}}^{2}+\left\|u_{t}\right\|_{L^{2}}^{2} & =-\int(u \cdot \nabla u) \cdot u_{t} d x+\int \theta e_{3} \cdot u_{t} d x \\
& \leq\|u\|_{L^{6}}\|\nabla u\|_{L^{3}}\left\|u_{t}\right\|_{L^{2}}+\|\theta\|_{L^{2}}\left\|u_{t}\right\|_{L^{2}} \\
& \leq C\left(\|\nabla u\|_{L^{2}}^{\frac{3}{2}}\|u\|_{H^{2}}^{\frac{1}{2}}\left\|u_{t}\right\|_{L^{2}}+\|\theta\|_{L^{2}}\left\|u_{t}\right\|_{L^{2}}\right) .
\end{aligned}
$$

On the other hand, $(1.1)_{1}$ can be rewritten as

$$
-\mu \Delta u+\nabla P=-u_{t}-u \cdot \nabla u+\theta e_{3} .
$$


By the $H^{2}$-theory of Stokes system, we derive that

$$
\mu\|u\|_{H^{2}} \leq C\left\|-u_{t}-u \cdot \nabla u+\theta e_{3}\right\|_{L^{2}} \leq C\left(\left\|u_{t}\right\|_{L^{2}}+\|\nabla u\|_{L^{2}}^{\frac{3}{2}}\|u\|_{H^{2}}^{\frac{1}{2}}+\|\theta\|_{L^{2}}\right)
$$

and thus

$$
\|u\|_{H^{2}} \leq \frac{C}{\mu}\left(\left\|u_{t}\right\|_{L^{2}}+\|\nabla u\|_{L^{2}}^{3}+\|\theta\|_{L^{2}}\right) .
$$

Inserting (2.7) into (2.6), we obtain

$$
\begin{aligned}
& \frac{\mu}{2} \frac{d}{d t}\|\nabla u(t)\|_{L^{2}}^{2}+\left\|u_{t}\right\|_{L^{2}}^{2} \\
& \quad \leq \frac{C}{\mu}\left(\|\nabla u\|_{L^{2}}^{\frac{3}{2}}\left\|u_{t}\right\|_{L^{2}}^{\frac{3}{2}}+\|\nabla u\|_{L^{2}}^{3}\left\|u_{t}\right\|_{L^{2}}+\|\nabla u\|_{L^{2}}^{\frac{3}{2}}\|\theta\|_{L^{2}}^{\frac{1}{2}}\left\|u_{t}\right\|_{L^{2}}\right)+C\|\theta\|_{L^{2}}\left\|u_{t}\right\|_{L^{2}} \\
& \quad \leq \frac{1}{2}\left\|u_{t}\right\|_{L^{2}}^{2}+C\left(\frac{1}{\mu^{4}}\|\nabla u\|_{L^{2}}^{6}+\frac{1}{\mu^{2}}\|\nabla u\|_{L^{2}}^{6}+\frac{1}{\mu^{2}}\|\nabla u\|_{L^{2}}^{3}\|\theta\|_{L^{2}}+\|\theta\|_{L^{2}}^{2}\right) \\
& \quad \leq \frac{1}{2}\left\|u_{t}\right\|_{L^{2}}^{2}+C\left(\frac{1}{\mu^{4}}\|\nabla u\|_{L^{2}}^{2}+\frac{1}{\mu^{2}}\|\nabla u\|_{L^{2}}^{2}+\|\nabla \theta\|_{L^{2}}^{2}\right) .
\end{aligned}
$$

Then, integrating $(2.8)$ over $(0, t)$, we have

$$
\mu\|\nabla u(t)\|_{L^{2}}^{2}+\int_{0}^{t}\left\|u_{t}(s)\right\|_{L^{2}}^{2} d s \leq C\left(1+\frac{1}{\mu^{5}}+\frac{1}{\mu^{3}}+\frac{1}{\kappa}\right),
$$

where we have used Lemma 2.1 and constant $C$ depending on $K_{1}$ and $K_{2}$.

Next, applying the operator $\nabla$ on both sides of temperature equation $(1.1)_{2}$ and multiplying by $\nabla \theta$, integrating the resulting equation over $\Omega$, we get

$$
\begin{aligned}
\frac{1}{2} \frac{d}{d t}\|\nabla \theta(t)\|_{L^{2}}^{2}+\kappa\left\|\nabla^{2} \theta\right\|_{L^{2}}^{2} & =-\int \nabla(u \cdot \nabla \theta) \cdot \nabla \theta d x \\
& \leq\|\nabla u\|_{L^{3}}\|\nabla \theta\|_{L^{6}}\|\nabla \theta\|_{L^{2}} \\
& \leq C\|u\|_{H^{2}}\left\|\nabla^{2} \theta\right\|_{L^{2}}\|\nabla \theta\|_{L^{2}} \\
& \leq \frac{\kappa}{2}\left\|\nabla^{2} \theta\right\|_{L^{2}}^{2}+\frac{C}{\kappa}\|u\|_{H^{2}}^{2}\|\nabla \theta\|_{L^{2}}^{2},
\end{aligned}
$$

integrating (2.10) over time $(0, t)$ and using Gronwall's inequality, which completes the proof of Lemma 2.2 .

Lemma 2.3 Under the conditions of Theorem 1.1, and suppose (1.5) is false. Then it holds that

$$
\sup _{0 \leq t \leq T}\left(\left\|u_{t}(t)\right\|_{L^{2}}^{2}+\left\|\theta_{t}(t)\right\|_{L^{2}}^{2}\right)+\int_{0}^{T}\left(\left\|\nabla u_{t}(s)\right\|_{L^{2}}^{2}+\left\|\nabla \theta_{t}(s)\right\|_{L^{2}}^{2}\right) d s \leq C,
$$

where constant $C$ depends on the initial data, $\mu, \kappa$, and some other known constants but is independent of $T$. 
Proof Taking the operator $\partial_{t}$ to $(1.1)_{1}$, multiplying the resulting equation by $u_{t}$ and integrating by parts, we have

$$
\begin{aligned}
\frac{1}{2} \frac{d}{d t}\left\|u_{t}(t)\right\|_{L^{2}}^{2}+\mu\left\|\nabla u_{t}\right\|_{L^{2}}^{2} & =-\int\left(u_{t} \cdot \nabla u\right) \cdot u_{t} d x+\int \theta_{t} e_{3} \cdot u_{t} d x \\
& \leq\|\nabla u\|_{L^{3}}\left\|u_{t}\right\|_{L^{6}}\left\|u_{t}\right\|_{L^{2}}+\left\|\theta_{t}\right\|_{L^{2}}\left\|u_{t}\right\|_{L^{2}} \\
& \leq C\left(\|u\|_{H^{2}}\left\|\nabla u_{t}\right\|_{L^{2}}\left\|u_{t}\right\|_{L^{2}}+\left\|\theta_{t}\right\|_{L^{2}}\left\|u_{t}\right\|_{L^{2}}\right) \\
& \leq \frac{\mu}{2}\left\|\nabla u_{t}\right\|_{L^{2}}^{2}+\frac{C}{\mu}\left(\|u\|_{H^{2}}^{2}\left\|u_{t}\right\|_{L^{2}}^{2}+\left\|\theta_{t}\right\|_{L^{2}}^{2}\right) .
\end{aligned}
$$

On the other hand, multiplying $(1.1)_{2}$ by $\theta_{t}$ and integrating the resulting equation over $\Omega$, we obtain

$$
\begin{aligned}
\frac{\kappa}{2} \frac{d}{d t}\|\nabla \theta(t)\|_{L^{2}}^{2}+\left\|\theta_{t}\right\|_{L^{2}}^{2} & =-\int(u \cdot \nabla \theta) \theta_{t} d x \leq\|u\|_{L^{\infty}}\|\nabla \theta\|_{L^{2}}\left\|\theta_{t}\right\|_{L^{2}} \\
& \leq \frac{1}{2}\left\|\theta_{t}\right\|_{L^{2}}^{2}+C\|u\|_{H^{2}}^{2}\|\nabla \theta\|_{L^{2}}^{2} .
\end{aligned}
$$

Then, integrating (2.12), (2.13) over $(0, t)$ and using Gronwall's inequality, we have

$$
\mu\left\|u_{t}\right\|_{L^{2}}^{2}+\kappa\|\nabla \theta\|_{L^{2}}^{2}+\int_{0}^{t}\left(\mu^{2}\left\|\nabla u_{t}(s)\right\|_{L^{2}}^{2}+\left\|\theta_{t}(s)\right\|_{L^{2}}^{2}\right) d s \leq C
$$

Next, applying the operator $\partial_{t}$ on both sides of temperature equation $(1.1)_{2}$ and multiplying by $\theta_{t}$, we get

$$
\begin{aligned}
\frac{1}{2} \frac{d}{d t}\left\|\theta_{t}(t)\right\|_{L^{2}}^{2}+\kappa\left\|\nabla \theta_{t}\right\|_{L^{2}}^{2} & =-\int\left(u_{t} \cdot \nabla \theta+u \cdot \nabla \theta_{t}\right) \theta_{t} d x \\
& \leq\left\|u_{t}\right\|_{L^{3}}\|\nabla \theta\|_{L^{6}}\left\|\theta_{t}\right\|_{L^{2}} \\
& \leq\left\|u_{t}\right\|_{H^{1}}^{2}+C\left\|\nabla^{2} \theta\right\|_{L^{2}}^{2}\left\|\theta_{t}\right\|_{L^{2}}^{2}
\end{aligned}
$$

integrating (2.15) over time $(0, t)$, using Gronwall's inequality, and taking (2.5), (2.14) into consideration, we obtain

$$
\left\|\theta_{t}(t)\right\|_{L^{2}}^{2}+\int_{0}^{t}\left\|\nabla \theta_{t}(s)\right\|_{L^{2}}^{2} d s \leq C,
$$

which completes the proof of Lemma 2.3 .

From Lemmas 2.1-2.3, we can see that Theorem 1.1 is proved.

\section{Global strong solution}

In this section, we prove the global existence and uniqueness of the strong solution. Assume that $T^{*}>0$ is the maximal existence time of the strong solution. We prove $T^{*}=+\infty$ by using contradiction arguments. If $T^{*}<+\infty$, our aim is to prove that (1.5) is not true under the conditions of Theorem 1.2, which is the desired contradiction. 
We define

$$
M(T)=\sup _{0 \leq t \leq T}\|\nabla u\|_{L^{2}}^{2}
$$

Proposition 3.1 Assume that the initial data satisfy the conditions in Theorem 1.2, and the local strong solution satisfies

$$
M(T) \leq 2 K_{1}, \quad(x, t) \in \Omega \times[0, T],
$$

then

$$
M(T) \leq \frac{3}{2} K_{1}, \quad(x, t) \in \Omega \times[0, T]
$$

provided that there exists $\varepsilon_{0}$ depending on $K_{1}, \mu, \kappa$, and some other known constants but independent of $T$ such that $\left\|u_{0}\right\|_{L^{2}}^{2}+\left\|\theta_{0}\right\|_{L^{2}}^{2} \leq \varepsilon_{0}$ is suitably small.

Lemma 3.2 Under the condition of Proposition 3.1, there exists $C^{*}$ depending on $K_{1}, \mu, \kappa$, and some other known constants but independent of $T$, it holds that

$$
M(T) \leq \frac{3}{2} K_{1}
$$

provided that $\varepsilon_{0} \leq C^{*}$.

Proof Recalling Lemma 2.2, from (2.8) we can see that

$$
\begin{aligned}
& \frac{\mu}{2} \frac{d}{d t}\|\nabla u(t)\|_{L^{2}}^{2}+\left\|u_{t}\right\|_{L^{2}}^{2} \\
& \quad \leq \frac{1}{2}\left\|u_{t}\right\|_{L^{2}}^{2}+C\left(\frac{1}{\mu^{4}}\|\nabla u\|_{L^{2}}^{6}+\frac{1}{\mu^{2}}\|\nabla u\|_{L^{2}}^{6}+\frac{1}{\mu^{2}}\|\nabla u\|_{L^{2}}^{3}\|\theta\|_{L^{2}}+\|\theta\|_{L^{2}}^{2}\right) \\
& \quad \leq \frac{1}{2}\left\|u_{t}\right\|_{L^{2}}^{2}+C\left(\frac{K_{1}^{2}}{\mu^{4}}\|\nabla u\|_{L^{2}}^{2}+\frac{K_{1}^{2}}{\mu^{2}}\|\nabla u\|_{L^{2}}^{2}+\|\nabla \theta\|_{L^{2}}^{2}\right) .
\end{aligned}
$$

Then, integrating (3.4) over $(0, t)$, we obtain

$$
\begin{aligned}
& \|\nabla u(t)\|_{L^{2}}^{2}+\frac{1}{\mu} \int_{0}^{t}\left\|u_{t}(s)\right\|_{L^{2}}^{2} d s \\
& \leq\left\|\nabla u_{0}\right\|_{L^{2}}^{2}+C\left(\frac{K_{1}^{2}}{\mu^{5}}+\frac{K_{1}^{2}}{\mu^{3}}\right) \int_{0}^{t}\|\nabla u(s)\|_{L^{2}}^{2} d s+\frac{C}{\mu} \int_{0}^{t}\|\nabla \theta(s)\|_{L^{2}}^{2} d s \\
& \leq\left\|\nabla u_{0}\right\|_{L^{2}}^{2}+\frac{C K_{1}^{2}}{\mu^{3}}\left(\frac{1}{\mu^{2}}+1\right)\left(\frac{1}{\mu}\left\|u_{0}\right\|_{L^{2}}^{2}+\frac{1}{\mu^{2} \kappa}\left\|\theta_{0}\right\|_{L^{2}}^{2}\right)+\frac{C}{\mu \kappa}\left\|\theta_{0}\right\|_{L^{2}}^{2},
\end{aligned}
$$

where we have used Lemma 2.1. Then we have

$$
\begin{aligned}
& \|\nabla u(t)\|_{L^{2}}^{2} \\
& \quad \leq\left\|\nabla u_{0}\right\|_{L^{2}}^{2}+\frac{C K_{1}^{2}}{\mu^{4}}\left(\frac{1}{\mu^{2}}+1\right)\left\|u_{0}\right\|_{L^{2}}^{2}+\frac{C K_{1}^{2}}{\mu^{5} \kappa}\left(\frac{1}{\mu^{2}}+1\right)\left\|\theta_{0}\right\|_{L^{2}}^{2}+\frac{C}{\mu \kappa}\left\|\theta_{0}\right\|_{L^{2}}^{2}
\end{aligned}
$$




$$
\begin{aligned}
& \leq\left\|\nabla u_{0}\right\|_{L^{2}}^{2}+\frac{C K_{1}^{2}}{\mu^{4}}\left(\frac{1}{\mu^{2}}+1\right)\left\|u_{0}\right\|_{L^{2}}^{2}+\frac{C}{\mu \kappa}\left(\frac{K_{1}^{2}}{\mu^{6}}+\frac{K_{1}^{2}}{\mu^{4}}+1\right)\left\|\theta_{0}\right\|_{L^{2}}^{2} \\
& \leq K_{1}+\frac{1}{2} K_{1}=\frac{3}{2} K_{1}
\end{aligned}
$$

provided $C^{*} \leq \frac{1}{2} K_{1} \delta^{-1}, \delta=\max \left\{\frac{C K_{1}^{2}}{\mu^{4}}\left(\frac{1}{\mu^{2}}+1\right), \frac{C}{\mu \kappa}\left(\frac{K_{1}^{2}}{\mu^{6}}+\frac{K_{1}^{2}}{\mu^{4}}+1\right)\right\}$. This completes the proof of Lemma 3.2.

Hence, from Theorem 1.1 and Proposition 3.1, we can see that Theorem 1.2 is proved provided

$$
\left\|u_{0}\right\|_{L^{2}}^{2}+\left\|\theta_{0}\right\|_{L^{2}}^{2} \leq \varepsilon_{0} \leq C^{*}
$$

\section{Decay estimates}

Finally, in this section, based on the global in time strong solution, we have the following exponential decay rate.

Lemma 4.1 Under the conditions of Theorem 1.2, we get that

$$
\int_{\Omega}\left(\theta^{2}+|\nabla u|^{2}\right) \leq C_{1} \exp \left\{-C_{2} t\right\}
$$

holds for any $t \in[0,+\infty)$, provided that

$$
\varepsilon_{0} \leq C^{*}
$$

for some positive constants $C_{1}, C_{2}$, and $C^{*}$ depending on $\mu, \kappa, K_{1}, K_{2}$, and some other known constants but independent of $t$.

Proof From (2.4) we can see that

$$
\frac{d}{d t}\|u(t)\|_{L^{2}}^{2}+\mu\|\nabla u\|_{L^{2}}^{2} \leq \frac{4}{\mu}\|\nabla \theta\|_{L^{2}}^{2} .
$$

Multiplying $\frac{2 C K_{1}^{2}}{\mu^{3}}\left(\frac{1}{\mu^{2}}+1\right)$ on both sides of inequality (4.2) and adding the resulting equation into (3.4), we obtain

$$
\begin{gathered}
\frac{d}{d t}\left(\frac{2 C K_{1}^{2}}{\mu^{3}}\left(\frac{1}{\mu^{2}}+1\right)\|u(t)\|_{L^{2}}^{2}+\frac{\mu}{2}\|\nabla u(t)\|_{L^{2}}^{2}\right) \\
\quad+\frac{2 C K_{1}^{2}}{\mu^{3}}\left(\frac{1}{\mu^{2}}+1\right)\|\nabla u(t)\|_{L^{2}}^{2}+\frac{1}{2}\left\|u_{t}\right\|_{L^{2}}^{2} \\
\leq C\left(\frac{8 K_{1}^{2}\left(1+\mu^{2}\right)}{\mu^{6}}+1\right)\|\nabla \theta(t)\|_{L^{2}}^{2} .
\end{gathered}
$$


Next, multiplying $2 C\left(\frac{8 K_{1}^{2}\left(1+\mu^{2}\right)}{\mu^{6} \kappa}+\frac{1}{\kappa}\right)$ on both sides of inequality (2.3) and adding the resulting equation into (4.3), we have

$$
\begin{aligned}
& \frac{d}{d t}\left(\frac{2 C K_{1}^{2}}{\mu^{3}}\left(\frac{1}{\mu^{2}}+1\right)\|u(t)\|_{L^{2}}^{2}+\frac{\mu}{2}\|\nabla u(t)\|_{L^{2}}^{2}+2 C\left(\frac{8 K_{1}^{2}\left(1+\mu^{2}\right)}{\mu^{6} \kappa}+\frac{1}{\kappa}\right)\|\theta(t)\|_{L^{2}}^{2}\right) \\
& \quad+\frac{2 C K_{1}^{2}}{\mu^{3}}\left(\frac{1}{\mu^{2}}+1\right)\|\nabla u(t)\|_{L^{2}}^{2}+C\left(\frac{8 K_{1}^{2}\left(1+\mu^{2}\right)}{\mu^{6} \kappa}+\frac{1}{\kappa}\right)\|\nabla \theta(t)\|_{L^{2}}^{2} \leq 0 .
\end{aligned}
$$

Taking

$$
Y(t)=\frac{2 C K_{1}^{2}}{\mu^{3}}\left(\frac{1}{\mu^{2}}+1\right)\|u(t)\|_{L^{2}}^{2}+\frac{\mu}{2}\|\nabla u(t)\|_{L^{2}}^{2}+2 C\left(\frac{8 K_{1}^{2}\left(1+\mu^{2}\right)}{\mu^{6} \kappa}+\frac{1}{\kappa}\right)\|\theta(t)\|_{L^{2}}^{2},
$$

it can be estimated as

$$
\begin{aligned}
Y(t) & \leq \frac{2 C K_{1}^{2}}{\mu^{3}}\left(\frac{1}{\mu^{2}}+1\right)\|\nabla u(t)\|_{L^{2}}^{2}+\frac{\mu}{2}\|\nabla u(t)\|_{L^{2}}^{2}+2 C\left(\frac{8 K_{1}^{2}\left(1+\mu^{2}\right)}{\mu^{6} \kappa}+\frac{1}{\kappa}\right)\|\nabla \theta(t)\|_{L^{2}}^{2} \\
& \leq\left(\frac{2 C K_{1}^{2}\left(1+\mu^{2}\right)}{\mu^{5}}+\frac{\mu}{2}\right)\|\nabla u(t)\|_{L^{2}}^{2}+2 C\left(\frac{8 K_{1}^{2}\left(1+\mu^{2}\right)}{\mu^{6} \kappa}+\frac{1}{\kappa}\right)\|\nabla \theta(t)\|_{L^{2}}^{2},
\end{aligned}
$$

where we have used the Poincaré inequality.

Then there exists a constant $M$ depending on $C, K_{1}, \mu, \kappa$ such that

$$
Y(t) \leq M\left(\frac{2 C K_{1}^{2}}{\mu^{3}}\left(\frac{1}{\mu^{2}}+1\right)\|\nabla u(t)\|_{L^{2}}^{2}+C\left(\frac{8 K_{1}^{2}\left(1+\mu^{2}\right)}{\mu^{6} \kappa}+\frac{1}{\kappa}\right)\|\nabla \theta(t)\|_{L^{2}}^{2}\right) .
$$

At last, from (4.4), we have

$$
Y^{\prime}(t)+\frac{1}{M} Y(t) \leq 0
$$

which deduces $Y(t) \leq Y(0) \exp \left\{-\frac{t}{M}\right\}$. This completes the proof of Theorem 1.3.

\section{Acknowledgements}

The authors would like to thank the anonymous referees for helpful suggestions and comments which improved our original paper, and the authors would also like to thank Journal of Inequalities and Applications for considering this paper to be published.

Funding

The authors' research was supported in part by Chinese National Natural Science Foundation under grants 11571232 and 11831011.

Availability of data and materials

Not applicable.

Competing interests

The authors declare that they have no competing interests.

\section{Authors' contributions}

The authors read and approved the final manuscript.

\section{Publisher's Note}

Springer Nature remains neutral with regard to jurisdictional claims in published maps and institutional affiliations. 


\section{References}

1. Abidi, H., Hmidi, T.: On the global well-posedness for Boussinesq system. J. Differ. Equ. 233(1), 199-220 (2007)

2. Brandolese, L., Schonbek, M.E.: Large time decay and growth for solutions of a viscous Boussinesq system. Trans. Am. Math. Soc. 364(10), 5057-5090 (2012)

3. Chae, D.: Global regularity for the 2D Boussinesq equations with partial viscosity terms. Adv. Math. 203(2), 497-513 (2006)

4. Chae, D., Kim, S.-K., Nam, H.-S.: Local existence and blow-up criterion of Hölder continuous solutions of the Boussinesq equations. Nagoya Math. J. 155, 55-80 (1999)

5. Chae, D., Nam, H.-S.: Local existence and blow-up criterion for the Boussinesq equations. Proc. R. Soc. Edinb., Sect. A 127(5), 935-946 (1997)

6. Danchin, R., Paicu, M.: Existence and uniqueness results for the Boussinesq system with data in Lorentz spaces. Physica D 237(10-12), 1444-1460 (2008)

7. Fan, J., Li, F.: Global strong solutions to the nonhomogeneous incompressible MHD equations in a bounded domain. Nonlinear Anal., Real World Appl. 46, 1-11 (2019)

8. Liu, X., Li, Y.: On the stability of global solutions to the 3D Boussinesq system. Nonlinear Anal. 95, 580-591 (2014)

9. Majda, A.: Introduction to PDEs and Waves for the Atmosphere and Ocean. Courant Lecture Notes in Mathematics, vol. 9. New York University, Courant Institute of Mathematical Sciences, New York (2003)

10. Majda, A.J., Bertozzi, A.L.: Vorticity and Incompressible Flow. Cambridge Texts in Applied Mathematics, vol. 27. Cambridge University Press, Cambridge (2002)

11. Qin, Y., Wang, Y., Su, X., Zhang, J.: Global existence of solutions for the three-dimensional Boussinesq system with anisotropic data. Discrete Contin. Dyn. Syst. 36(3), 1563-1581 (2016)

12. Sawada, O., Taniuchi, Y.: On the Boussinesq flow with nondecaying initial data. Funkc. Ekvacioj 47(2), $225-250$ (2004)

13. Wen, H., Zhu, C.: Global solutions to the three-dimensional full compressible Navier-Stokes equations with vacuum at infinity in some classes of large data. SIAM J. Math. Anal. 49(1), 162-221 (2017)

14. Wen, Z., Ye, Z:: On the global existence of strong solution to the $3 D$ damped Boussinesq equations with zero thermal diffusion. Z. Anal. Anwend. 37(3), 341-348 (2018)

15. Ye, Z.: Global regularity for a 3D Boussinesq model without thermal diffusion. Z. Angew. Math. Phys. 68(4), Article ID $83(2017)$

16. Ye, Z:: On global well-posedness for the 3D Boussinesq equations with fractional partial dissipation. Appl. Math. Lett. 90, 1-7 (2019)

17. Ye, Z: An alternative approach to global regularity for the $2 d$ Euler-Boussinesq equations with critical dissipation. Nonlinear Anal. 190, 111591 (2020)

\section{Submit your manuscript to a SpringerOpen ${ }^{0}$ journal and benefit from:}

- Convenient online submission

- Rigorous peer review

- Open access: articles freely available online

- High visibility within the field

- Retaining the copyright to your article

Submit your next manuscript at $>$ springeropen.com 